PAPER

\title{
The vestibular control of balance after stroke
}

\section{J F Marsden, E D Playford, B L Day}

See Editorial Commentary, p 621

J Neurol Neurosurg Psychiatry 2005;76:670-678. doi: 10.1136/jnnp.2004.046565

Objectives: To examine vestibular control of balance in those who recovered the ability to stand after middle cerebral artery (MCA) stroke.

Methods: Sixteen patients with MCA stroke were compared with 10 age matched controls. Two additional

See end of article for authors' affiliations

......................

Correspondence to:

Dr J Marsden, MRC

Human Movement Group,

Sobell Department of

Movement Disorders and

Motor Neuroscience,

Institute of Neurology,

Queen Square, London

WCIN 3BG, UK:

j.marsden@ion.ucl.ac.uk

Received 1 June 2004

In revised form

19 July 2004

Accepted 22 July 2004 patients were studied with isolated corticospinal tract lesions, one each at the level of the pons and medulla. Vestibular evoked postural responses were obtained using galvanic vestibular stimulation (GVS) while patients stood with their eyes closed and head facing forwards, equally loading both legs. The GVS response was characterised by measuring the amplitude of the stimulus evoked lateral forces acting through each leg and the lateral displacement of the axial skeleton.

Results: Lateral displacement and net lateral force following GVS were significantly larger after stroke. Unlike controls, the lateral forces in the stroke group were asymmetrical, being enhanced on the side of the non-paretic limb and small on the side of the paretic limb. The degree of GVS evoked asymmetry correlated with corticospinal damage assessed using transcranial magnetic stimulation. A similar asymmetrical response was seen in the patient with the pontine lesion but not the patient with the medullary lesion.

Conclusions: MCA stroke may disrupt corticobulbar projections to brainstem output pathways involved in vestibular control of balance. These projections are either collaterals of the corticospinal tract or lie close to that tract and terminate in the pons/upper medulla. This hypothesis accounts for the association between corticospinal tract damage and GVS response asymmetry, and the lack of GVS evoked asymmetry with corticospinal lesions below the rostral medulla.
$\mathrm{D}$ eficits in posture and balance are often seen after a middle cerebral artery (MCA) stroke. This is most apparent during the acute phase following a stroke, when patients may be unable to stand or walk, but chronic deficits are also seen that may contribute to falls and immobility. For example, standing posture is often asymmetrical, with less weight being taken on the paretic side contralateral to the lesion. ${ }^{12}$ Asymmetries are also seen in response to postural perturbations, or in association with self initiated upper or lower limb movements, with muscle responses in the paretic lower limbs being delayed in onset or decreased in amplitude. ${ }^{3-11}$ However, the pathophysiological mechanisms underlying these asymmetries remain unclear.

In our present study, we have investigated the longterm effects of an MCA stroke on a specific balance process. This is a mechanism that produces whole body adjustments in response to changes in vestibular information, and which contributes to the balance process during stance. It is possible to activate this pathway artificially by stimulating vestibular nerve afferents using galvanic vestibular stimulation (GVS). The stimulus modulates the firing rate of vestibular nerve afferents, with the application of an anode or a cathode decreasing or increasing their firing rate, ${ }^{12-14}$ respectively, resulting in a reproducible postural sway. In healthy subjects standing with their eyes closed and their head facing forwards, the stimulus produces a lateral sway of the body, which culminates in a tilted and bent posture. ${ }^{15}$ It has been suggested that the stimulus evoked response is mediated via signals descending in the vestibulospinal and/or the reticulospinal tracts, ${ }^{16}{ }^{17}$ which in turn arise from the vestibular and reticular nuclei within the brainstem.

One advantageous feature of the GVS technique is that it is possible to evoke a bilateral response by stimulating vestibular afferents on one side only. ${ }^{18} 19$ This provides a means of identifying any asymmetries that may exist in the response pattern. It also offers an opportunity for distinguishing between an asymmetry arising from an abnormality in the processing of sensory information from one ear and an abnormality in the motor control of one side of the body. A lateralised sensory disturbance might be expected to produce a response deficit in both legs when stimulating one ear but not the other. In contrast, a lateralised motor disturbance might be expected to produce a response deficit in one leg and not the other, irrespective of which ear is stimulated. GVS also enables a response direction asymmetry to be tested by taking advantage of the fact that reversing the polarity of stimulation causes subjects to respond in a diametrically opposite direction. This form of asymmetry might be expected to produce a response deficit when the response is in one direction-for example, towards the lesioned sidebut not the other.

Therefore, the experiment involved measuring the response of both legs to four types of monaural vestibular stimulation (two polarities of stimulation on two sides), in people with MCA stroke. We also examined the relation between corticospinal disruption and abnormalities of the GVS evoked response by measuring the short latency muscle response to single pulse transcranial magnetic stimulation (TMS). Previous work has shown that TMS produces muscle responses that are delayed in onset and/or have reduced amplitude after stroke. ${ }^{20}$ Finally, in two people we measured how the response to vestibular stimulation and TMS was affected by relatively discrete lesions to the corticospinal tract at the level of the pons or medulla.

Abbreviations: EMG, electromyography; GVS, galvanic vestibular stimulation; MCA, middle cerebral artery; MEP, motor evoked potential; $M R I$, magnetic resonance imaging; TMS, transcranial magnetic stimulation 


\begin{tabular}{|c|c|c|}
\hline & \multicolumn{2}{|c|}{ Side of stimulation } \\
\hline & $\begin{array}{l}\text { Ipsilateral to } \\
\text { lesion }\end{array}$ & $\begin{array}{l}\text { Contralateral to } \\
\text { lesion }\end{array}$ \\
\hline $\begin{array}{l}\text { Sway direction } \\
\text { Ipsilateral to lesion } \\
\text { Contralateral to lesion }\end{array}$ & $\begin{array}{l}\text { Anode } \\
\text { Cathode }\end{array}$ & $\begin{array}{l}\text { Cathode } \\
\text { Anode }\end{array}$ \\
\hline \multicolumn{3}{|c|}{$\begin{array}{l}\text { The table indicates the relationship between the site and polarity of the } \\
\text { active electrode and the stimulus evoked sway direction. The site of } \\
\text { stimulation and the stimulus evoked sway direction are given relative to } \\
\text { the side of the lesion. }\end{array}$} \\
\hline
\end{tabular}

\section{METHODS}

\section{Clinical details}

Patients with an MCA stroke were recruited from the National Hospital for Neurology and Neurosurgery, London, UK, and from a local stroke self help group. Subjects were recruited if they were able to walk $10 \mathrm{~m}$ independently, with or without the use of a gait aid, at the time of our study, although all had been unable to stand or walk independently in the acute stages after the stroke. Exclusion criteria were the presence of a previous stroke or other neurological or orthopaedic impairments. Age and sex matched healthy subjects with no history of neurological or orthopaedic impairments were recruited as controls. In addition, we assessed two subjects who had discrete lesions predominately affecting the pyramidal tract, one within the pons and the other in the medulla, to elucidate further the role of corticofugal projections to brainstem pathways. All subjects participated with informed consent and the approval of the local ethics committee according to the Declaration of Helsinki.

\section{Galvanic vestibular stimulation procedure}

Subjects stood barefoot with each foot on a separate force plate with a $5 \mathrm{~cm}$ gap between the medial borders of the feet. A visual display $1 \mathrm{~m}$ in front of the subjects gave feedback in $5 \%$ steps about the percentage of body weight being taken by the right leg. After a warning tone, a central light appeared above the feedback display that indicated the point when the legs took equal weight. Once subjects had achieved equal lower limb weight bearing with the aid of the feedback display, they were instructed to close their eyes and the trial was started. After a 0.5 to two second random delay, data collection began. After a three second baseline period, a three second, 1.0 mA monaural galvanic vestibular stimulus was given, which was then followed by a further three second period. The stimulus was applied using $2.5 \mathrm{~cm}$ diameter electrodes; the active electrode was placed on the mastoid process with an indifferent electrode on the back at the level of Tl spinous process (PALS plus; Nidd Valley Medical, Knaresborough, North Yorkshire, UK). There were four stimulus conditions. The active electrode on the mastoid process could either be on the same or opposite side to the lesion and, by altering the stimulus polarity, it could produce a sway either towards or away from the side of the lesion (table 1). The order of stimulus presentation was randomised, with 10 stimuli being given for each condition.

Axial displacement was measured using a three dimensional motion analysis system (CODA MPX30 system; Charnwood Dynamics, Rothley, Leicestershire, UK) via markers attached to a helmet, semirigid belt, and to the back at the level of the C7 and L3 spinous processes. Lateral reaction forces were obtained from the force plates under each foot (Kistler 9281B, left leg; Kistler 9287, right leg). Throughout the experiment, subjects wore a safety harness, which was attached to an overhead gantry capable of low friction movement in the horizontal plane.

\section{Single pulse, transcranial magnetic stimulation procedure}

The motor evoked potential (MEP) elicited in the preactivated tibialis anterior muscle was assessed. Subjects sat with their foot plantigrade in a custom made manipulandum. The axis of ankle dorsi/plantarflexion was collinear with the axis of rotation of the manipulandum. Subjects isometrically dorsiflexed the foot to a constant torque of $0.5 \mathrm{Nm}$, as measured via a strain gauge. Visual feedback of ankle torque and the required target value was provided on an oscilloscope. Muscle activity from the gastrocnemius was recorded via surface electromyography (EMG) to ensure that no cocontraction occurred during this task.

Single pulse TMS was given via a figure of eight coil placed on the vertex, with the current acting in an antero-posterior direction. Initially, the threshold for stimulation (defined as the value that gave a response in three of five stimuli) was determined on the non-paretic side during isometric dorsiflexion. Five stimuli were then recorded at $\times 1.5$ motor threshold. After this, the paretic limb was assessed using the same coil position and stimulus intensities as for the nonparetic side with the ankle in an identical position, dorsiflexing to the same constant torque. The maximal motor response was then elicited in the tibialis anterior by stimulating the common peroneal nerve at the level of the head of the fibula, with the tibialis anterior muscle relaxed. Three stimuli were recorded for the left and the right legs. Tibialis anterior muscle responses were recorded via surface EMG (MT8 MIE; Medical Research, Leeds, UK). Signals were sampled at $2 \mathrm{kHz}$ after amplification.

\section{Clinical measures}

The time taken and the number of paces required to walk $10 \mathrm{~m}$ at the patients' normal pace were recorded. Lower limb muscle strength was manually tested and graded on a 0-5 ordinal scale. ${ }^{22}$ The summed scores for the hip flexors, abductors, and extensors, the knee extensors and flexors, and the ankle dorsiflexors and plantarflexors were calculated, giving a total maximum score of 35. Distal lower limb sensation was tested using the Rivermead assessment of somatosensory performance (RASP; Thames Valley Test Company Ltd, Bury St Edmunds, UK). This assessed light touch on the plantar and dorsal aspects of the foot (test 2) and proprioceptive awareness at the ankle and hallux (test 7).

\section{Measurements}

The results for patients with right and left sided lesions were combined. The leg contralateral to the lesion was defined as the paretic side, whereas the leg ipsilateral to the lesion was defined as the non-paretic side. To account for the different lesion sides in the patients, the control subjects were randomly assigned to two groups. For six control subjects the left leg was compared with the paretic leg of the stroke subjects, whereas in the remaining four control subjects the right leg was compared with the paretic leg of the stroke patients.

\section{Galvanic vestibular stimulation}

In response to GVS, all subjects swayed towards the anode or away from the cathode. In light of this, the type of stimulus was defined according to whether the active electrode was ipsilateral or contralateral to the lesion, and whether the stimulus caused a sway towards or away from the lesion. Lateral reaction forces were defined as positive if they occurred in the direction of sway. 


\begin{tabular}{|c|c|c|c|c|c|c|}
\hline Subject & Age (years) & Sex & Time since lesion (months) & Lesion location & GVS Al & TMS delay (ms) \\
\hline 1 & 47 & M & 90 & L MCA infarct, fronto-temporo-parietal & -1 & NA \\
\hline 2 & 19 & M & 22 & R MCA haemorrhage, fronto-temporo-parietal & -1 & NA \\
\hline 3 & 76 & M & 32 & $\mathrm{~L}$ thalamic and IC infarct & -1 & 15.5 \\
\hline 4 & 65 & M & 12 & $\begin{array}{l}\text { L anterior artery and anterior MCA infarct, fronto- } \\
\text { parietal }\end{array}$ & -1 & 12.5 \\
\hline 5 & 62 & $\mathrm{~F}$ & 25 & $\mathrm{R}$ anterior and posterior limbs of IC and caudate & -0.70 & 15 \\
\hline 6 & 66 & M & 24 & $R$ anterior watershed territory infarct & -0.64 & 7 \\
\hline 7 & 33 & M & 29 & L MCA, fronto-parietal & -0.57 & 5 \\
\hline 8 & 55 & M & 24 & R MCA, temporo-parietal infarct & -0.56 & 7 \\
\hline 9 & 46 & M & 28 & $\begin{array}{l}\mathrm{L} \text { focal lesion at the junction of the thalamus and the } \\
\text { posterior limb of the IC }\end{array}$ & -0.46 & 7.5 \\
\hline 10 & 40 & $\mathrm{~F}$ & 12 & $\begin{array}{l}\text { Leff lentiform/head of caudate and anterior and } \\
\text { posterior IC infarct }\end{array}$ & -0.41 & 3.5 \\
\hline 11 & 66 & M & 25 & $\begin{array}{l}\mathrm{R} \text { caudate nucleus infarct extending into the corona } \\
\text { radiate }\end{array}$ & -0.35 & 10 \\
\hline 12 & 68 & M & 19 & R posterior limb IC & -0.32 & 6.5 \\
\hline 13 & 53 & $\mathrm{~F}$ & 46 & $\begin{array}{l}\text { R MCA temporo-frontal infarct extending into basal } \\
\text { ganglia }\end{array}$ & -0.28 & 4 \\
\hline 14 & 53 & M & 24 & $\begin{array}{l}\mathrm{R} \text { posterior right lentiform nucleus and the adjacent } \\
\text { IC infarct }\end{array}$ & -0.25 & 7 \\
\hline 15 & 55 & M & 37 & R MCA & -0.21 & 2.5 \\
\hline 16 & 70 & M & 45 & $\begin{array}{l}R \text { caudate and putamen infarction with secondary } \\
\text { degeneration of the right cerebral peduncles and pons }\end{array}$ & 0.23 & -3.5 \\
\hline
\end{tabular}

For each trial, the mediolateral postural sway was measured over the baseline period. This was defined as the standard deviation of the mediolateral velocity of the C7 marker following $20 \mathrm{~Hz}$ low pass filtering. The lateral reaction forces and the lateral displacement of the axial markers were used to measure the GVS evoked postural response. Any baseline drift in the axial displacement or reaction force in each trial was removed by subtracting the slope over the baseline period, estimated by a least squares linear regression, from the complete trace. Secondary analysis was then performed on the average of the 10 trials for each condition.

The initial lateral force response was characterised by determining the change in impulse (force $\times$ time) from 320$500 \mathrm{~ms}$ after stimulus onset. The average lateral displacement from 520-700 ms post stimulus onset of the head, C7, and pelvic markers were calculated (see grey bars in fig 1A). The initial lateral reaction force response averaged across all stimulus conditions was also used to calculate an asymmetry index, which was defined as: $(\mathrm{P}-\mathrm{NP}) /(\mathrm{P}+\mathrm{NP})$, where $\mathrm{P}$ and NP refer to the paretic and non-paretic leg responses, respectively. In some subjects with stroke, the paretic leg could generate force in the opposite direction to the direction of sway, this would generate an asymmetry index more negative than -1 . In these cases $(n=4)$, the limit was set to -1 . Therefore, the index was bounded from +1 to -1 , where
0 indicates that both legs contribute equally to the postural sway.

\section{Single pulse transcranial magnetic stimulation}

The average response to cortical or peripheral nerve stimulation was first calculated. The cortically elicited MEP was then rectified and normalised by the peak to peak amplitude of the maximal motor response obtained with the peripheral nerve stimulus. The onset and offset of the normalised MEP was defined as the time the response exceeded or fell below the mean baseline level \pm 2 SD for at least $10 \mathrm{~ms}$. The mean amplitude of the response was calculated between these limits.

The asymmetry index was defined as: $(\mathrm{P}-\mathrm{NP}) /(\mathrm{P}+\mathrm{NP})$, where NP indicates the amplitude response of the nonparetic leg and $\mathrm{P}$ indicates the amplitude of the paretic leg. As with the GVS asymmetry index, this is a bounded measure taking values from -1 to 1 , where 0 indicates symmetrical responses between the two limbs.

\section{Statistical analysis}

The parameters measured were analysed using a between groups general linear model in SPSS (version 11). The response to the four GVS conditions was analysed using the stimulus side relative to the lesion (two levels-ipsilateral or contralateral) and the sway direction relative to the lesion

Table 3 Group clinical details

\begin{tabular}{|c|c|c|c|c|c|c|c|c|c|}
\hline \multirow[b]{2}{*}{ Group } & \multirow[b]{2}{*}{$M / F$} & \multirow[b]{2}{*}{ Age (years) } & \multirow[b]{2}{*}{ Mass (kg) } & \multirow[b]{2}{*}{ Height (m) } & \multicolumn{2}{|c|}{$10 \mathrm{~m}$ timed walk } & \multirow{2}{*}{$\begin{array}{l}\text { Muscle } \\
\text { strength NP } \\
(\mathrm{n}=35)\end{array}$} & \multirow{2}{*}{$\begin{array}{l}\text { Muscle } \\
\text { strength P } \\
(n=35)\end{array}$} & \multirow{2}{*}{$\begin{array}{l}\text { Sensation test } 7 \text { P } \\
(n=24)\end{array}$} \\
\hline & & & & & Time (s) & Steps & & & \\
\hline R stroke & $8 / 2$ & $57.0(13.9)$ & 72.9 (12.4) & $1.65(0.26)$ & $24(2.3)$ & $22.4(15.4)$ & $23.5(8.5)$ & $34(2)$ & $25(8.5)$ \\
\hline L stroke & $5 / 1$ & $51.2(16.2)$ & $79.6(16.7)$ & $1.69(0.12)$ & $23(5)$ & $23.5(21.6)$ & $24.6(11.8)$ & $33.5(2.5)$ & $26.5(4.8)$ \\
\hline Controls & $7 / 3$ & $57.3(13.2)$ & 85.7 (15.3) & $1.72(0.10)$ & $24(0) \dagger$ & $7.8(0.8)^{*}$ & $14.3(1.8)^{*}$ & $35(0) \dagger$ & $35(0) \dagger$ \\
\hline
\end{tabular}

The mean (SD) is presented, except for the measures of muscle strength and sensory function, where the median and interquartile range is given. The total scores indicate the maximum score possible for this test. NP and P indicate the scores for the non-paretic and the paretic legs, respectively.

*The stroke group (left and right sided lesions combined) was significantly different from the control group, as measured using a two tailed unpaired $t$ test. $†$ The stroke group was significantly different from the control group, as measured using a Mann-Whitney test.

$L$, left; R, right. 
A

Stroke

Control
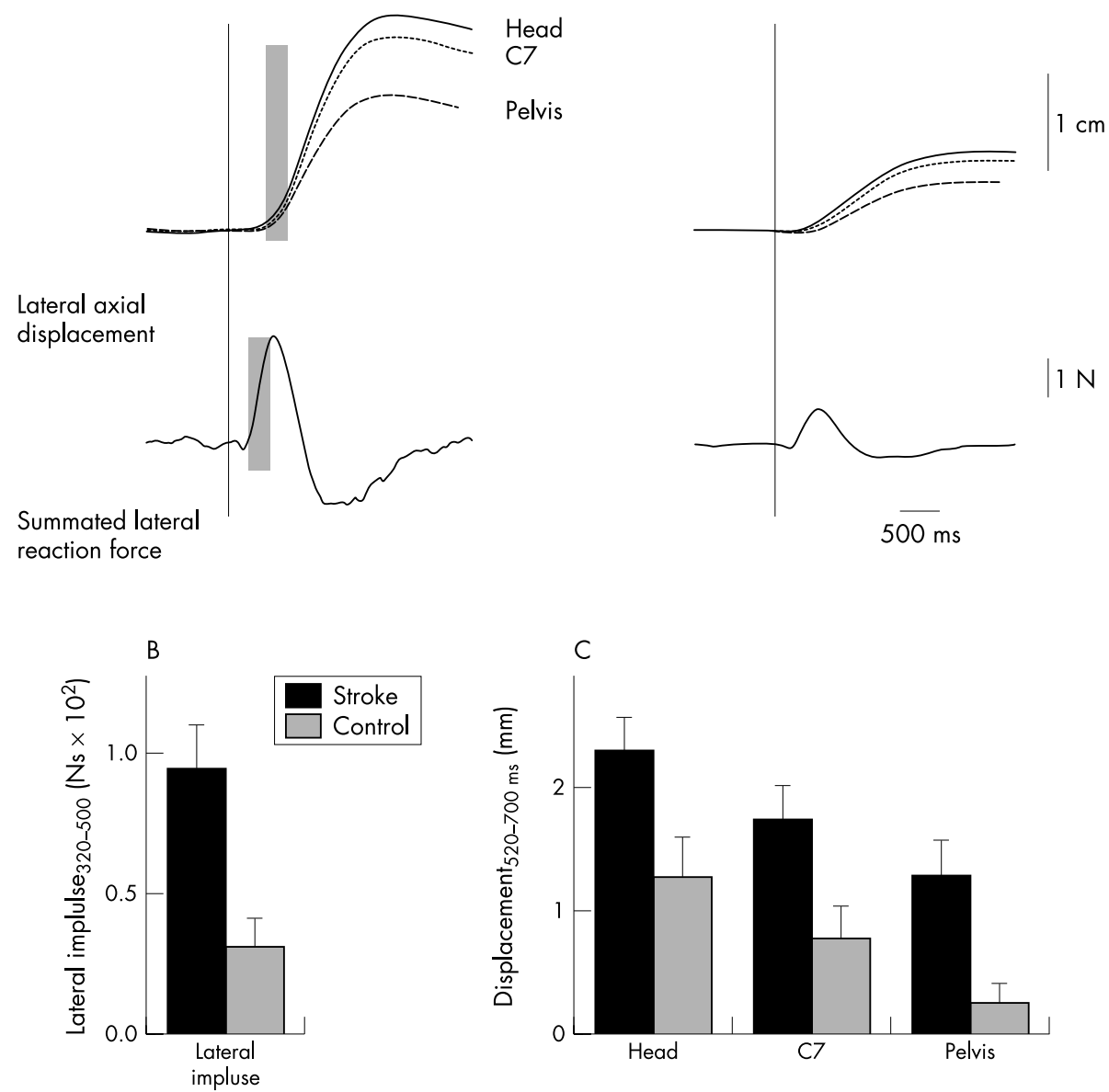

Figure 1 Net response to galvanic vestibular stimulation. (A) The grand average responses averaged across all stimulus conditions for the stroke group ( $\mathrm{n}=16$, left side) and the control group ( $\mathrm{n}=10$, right side). In this and subsequent figures the vertical line indicates the time of stimulus onset, and upward going lateral forces indicate forces acting on the body in the direction of sway. The grey bars indicate the period over which the initial change in impulse and lateral displacement were measured. (B) The mean (SEM) net lateral impulse measured from 320-500 ms after stimulus onset. (C) The mean (SEM) lateral axial displacement measured from 520-700 ms after stimulus onset.

(two levels-towards or away from the side of the lesion) as factors. When assessing the forces produced by either leg an additional factor of leg (two levels-paretic or non-paretic) was included. The TMS amplitude and onset were assessed using the factor leg. Pairwise comparisons between groups were made using a two tailed unrelated $t$ test or a MannWhitney test, as indicated. Relations between variables measured were calculated using linear regression. Significance was taken as $\mathrm{p}<0.05$.

\section{RESULTS}

\section{Clinical details}

An ischaemic stroke occurred in 15 patients and a haemorrhagic stroke occurred in one patient. Patients were seen on average 30.9 (SEM, 4.6) months after stroke onset and had a mean age of 54.6 (SEM, 3.8) years. Both cortical and subcortical lesions were present as defined on computed tomography or magnetic resonance imaging (MRI) scans (table 2). There was no difference between the stroke and control groups in terms of age (two tailed unpaired $t$ test, $\mathrm{t}(24)= \pm 0.37 ; \quad \mathrm{p}>0.05), \quad$ mass $(\mathrm{t}(24)= \pm 1.7 ;$ $\mathrm{p}>0.05)$, or height $(\mathrm{t}(24)= \pm 0.6$; $\mathrm{p}>0.05$; table 3$)$.

All patients had recovered the ability to walk in the period one to three months after stroke, although as indicated in table 3, deficits in walking speed were still apparent. The patients with stroke also presented with lower limb weakness and sensory deficits (table 3 ) compared with the control subjects. No patient had signs of spatial neglect as assessed by a psychologist at the time of initial admission.

\section{Response to GVS}

The legs were equally loaded over the baseline period. The paretic leg was loaded by $48.9 \%$ of body weight in the stroke group (SEM, 1.1\%) and 50.7\% (SEM, 0.5\%) in the control group $(\mathrm{t}(24)=1.3 ; \mathrm{p}>0.05)$. There was a tendency for stroke patients to shift their weight slowly on to the nonparetic leg over the baseline period. However, this effect was small, and there was no significant difference in the average load taken by the paretic limb over the first $200 \mathrm{~ms}$ and the last $200 \mathrm{~ms}$ of the baseline period. The degree of mediolateral postural sway over the baseline period, as measured from the C7 marker, was larger in the stroke group (two tailed $t$ test, $\mathrm{t}(24)= \pm 2.8 ; \mathrm{p}<0.05)$.

\section{Net response characteristics}

In response to GVS, all subjects swayed away from the active electrode if it was a cathode or towards the active electrode if it was an anode. The displacement of the axial markers following GVS was significantly larger in the stroke group (group factor: head, $\mathrm{F}(1,24)=5.9 ; \mathrm{p}<0.05 ; \quad \mathrm{C} 7$, $\mathrm{F}(1,24)=5.7 ; \mathrm{p}<0.05$; pelvis, $\mathrm{F}(1,24)=6.8 ; \mathrm{p}<0.05)$. The total summated lateral reaction force acting on the body 
A Stroke

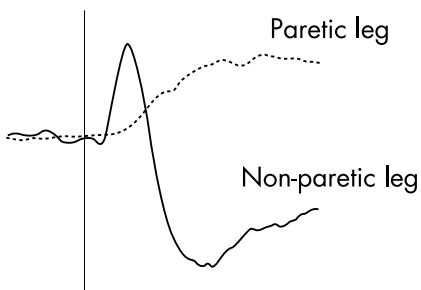
each leg
Lateral forces through

Control

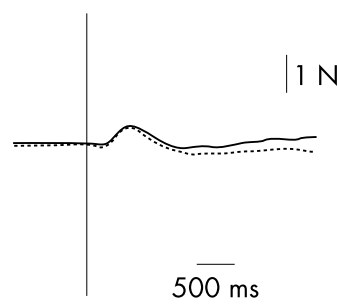

$11 \mathrm{~N}$

B

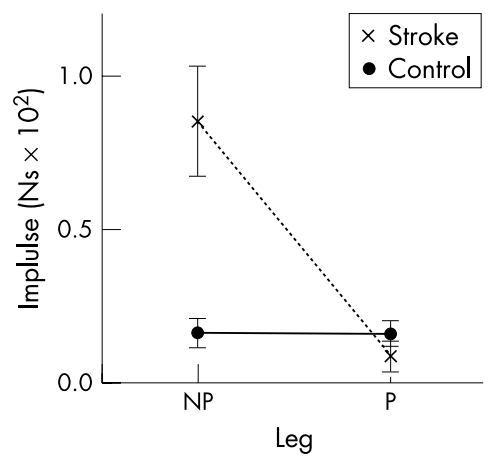

Figure 2 Differences between groups in the galvanic vestibular stimulation (GVS) evoked lateral forces acting through each leg. (A) The grand average response of the individual lateral reaction forces is shown averaged across all stimulus conditions for the stroke $(n=16)$ and control $(n=10)$ groups. (B) Mean (SEM) lateral impulse acting through each leg $320-500 \mathrm{~ms}$ after stimulus onset. NP, non-paretic; $P$, paretic.

(that is, the sum of the left and right leg responses) was also higher in the stroke group (group factor: $\mathrm{F}(1,24)=10.4$; $\mathrm{p}<0.005)$. However, there was no significant effect of stimulus side or sway direction on either of these measures. Figure 1 summarises the effect of GVS on the summated lateral reaction forces and axial displacement after averaging across all stimulus conditions.

\section{Individual forces}

The GVS evoked lateral forces acting through each leg were asymmetrical after stroke. The initial lateral impulse (measured from 320-500 ms after stimulus onset) was higher on the non-paretic side than on the paretic side, whereas such consistent asymmetries were not seen in the control group $($ group $\times$ leg interaction, $(\mathrm{F}(1,24)=6.87 ; \mathrm{p}<0.05$; fig $2 \mathrm{~A}$ and $\mathrm{B})$. There were no effects of stimulus side, sway direction, or other interactions. This indicated that the stroke group's asymmetrical response was present regardless of the stimulus condition. Therefore, further post hoc analysis was performed on the lateral forces after averaging across stimulus conditions. This analysis revealed that the amplitude of the response was significantly higher in the nonparetic leg compared with controls (unpaired $t$ test, $\mathrm{t}(24)= \pm 3.0 ; \mathrm{p}<0.01)$, whereas there was no difference in the size of the response in the paretic leg compared with the control group (unpaired t test, $\mathrm{t}(24)= \pm 0.7 ; \mathrm{p}>0.05$; fig 2B).

The asymmetry in the response between the two legs was highlighted by a significantly larger mean (SEM) asymmetry index of $-0.53(0.09$; table 2$)$ for the stroke group compared with $0.04(0.13)$ for the control group (unpaired t test, $\mathrm{t}(24)= \pm 3.8 ; \mathrm{p}<0.001)$, where zero indicates perfect symmetry between the two sides.
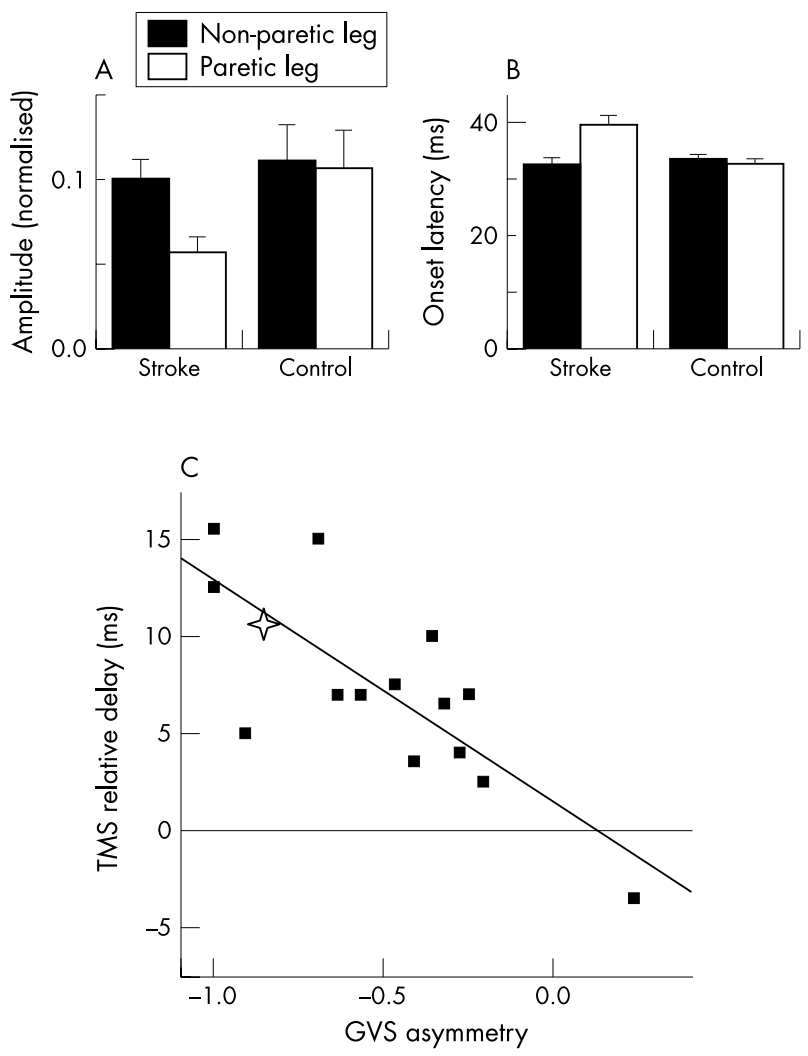

Figure 3 Response to transcranial magnetic stimulation. The mean (SEM) (A) Motor evoked potential amplitude and (B) onset latency in response to transcranial magnetic stimulation (TMS). (C) Correlation between the relative delay in the TMS and the galvanic vestibular stimulation (GVS) asymmetry index $\left(R^{2}=0.60\right)$. The star symbol indicates data from one patient who had suffered a discrete anterior pontine stroke.

\section{Response to single pulse TMS}

Only 14 patients in the stroke group participated in this part of the experiment. Two were unable to participate because of a history of epilepsy and the presence of intracranial metal.

The mean (SEM) stimulation threshold in terms of maximum output of the stimulator was $36.8 \%$ (1.9\%) for the stroke subjects and $34.0 \%(2.9 \%)$ for the control subjects. The amplitude of the response revealed a significant group $x$ leg interaction $(\mathrm{F}(1,22)=5.0 ; \mathrm{p}<0.05)$. Post hoc analysis showed no difference between groups on the non-paretic side (unpaired test, $\mathrm{t}(24)= \pm 0.57 ; \mathrm{p}>0.05)$, whereas the response was significantly smaller for the stroke group on the paretic side (unpaired $\mathrm{t}$ test, $\mathrm{t}(24)= \pm 2.7 ; \mathrm{p}<0.05$; fig $3 \mathrm{~A}$ ). The asymmetry index of response amplitude was different for the two groups (stroke: mean, -0.29 ; SEM, 0.06; control: -0.03 ; SEM, 0.09; unpaired $t$ test, $(\mathrm{t}(22)=2.7$; $\mathrm{p}<0.05)$.

In the stroke group, the TMS response was delayed on the paretic side by $7.1 \mathrm{~ms}$ (SEM, 1.3; table 2), compared with $-0.85 \mathrm{~ms}$ (SEM, 0.5) for the control subjects (unpaired two tailed $t$ test, $\mathrm{t}(22)=4.5 ; \mathrm{p}<0.0005$; fig $3 \mathrm{~B})$.

\section{Correlations between the responses seen after GVS and TMS}

There was a significant correlation between the asymmetry index seen after GVS and the relative delay in the MEP after TMS $\left(R^{2}=0.61 ; \mathrm{p}<0.001\right.$; fig $\left.3 \mathrm{C}\right)$. The linear regression model was not significantly improved by using measures of TMS response amplitude asymmetry, muscle strength, or hemiplegic limb sensory deficit as predictors. Highly 

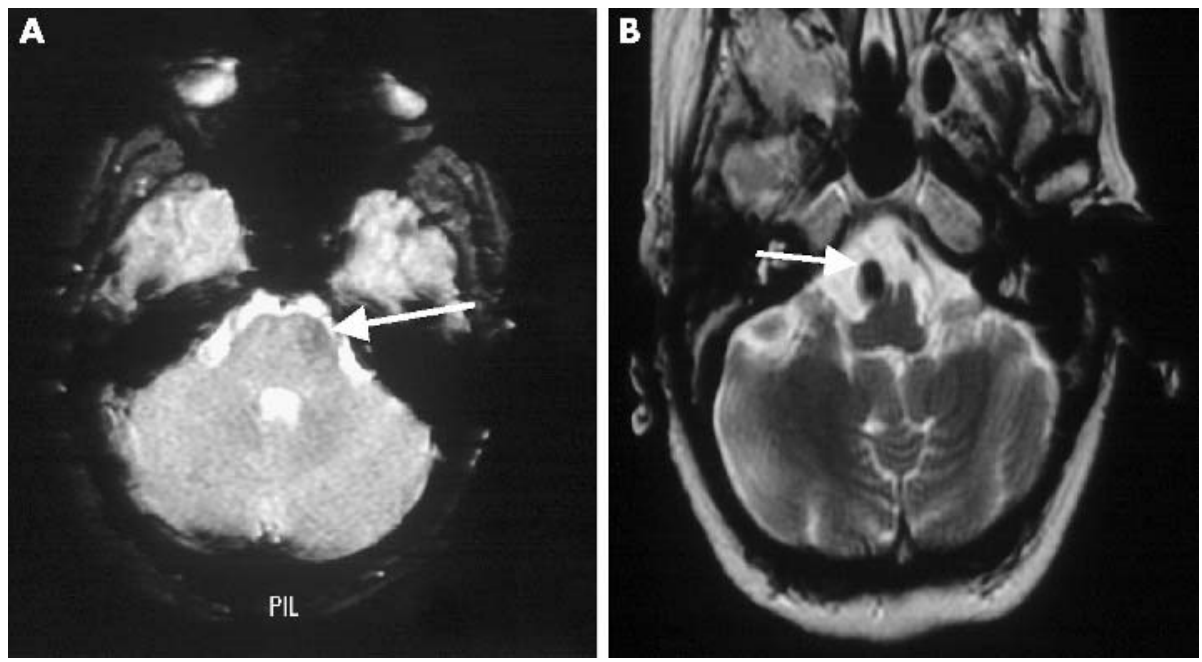

Figure 4 Lesion location in two patients with discrete corticospinal lesions. (A) Horizontal magnetic resonance imaging (MRI) scan indicating a left anterior pontine lacunar infarct in subject 1 (arrow). (B) Horizontal MRI of the medulla indicating a right vertebral artery aneurysm compressing the right pyramid in subject 2 (arrow).

asymmetrical responses, with a GVS asymmetry index more negative than -0.8 , were seen even when clinical testing of proprioception at the ankle and hallux was intact. There was no observed effect of lesion location (cortical versus subcortical) or period since stroke on the response asymmetry seen with GVS.

\section{Deficits in the GVS response after discrete pyramidal lesions}

To investigate further the relation between corticospinal tract damage and the asymmetrical response to GVS, two additional patients with lesions predominately affecting the pyramidal tract were assessed. Importantly, these pyramidal tract lesions were either rostral or caudal to the vestibular nuclei (fig 4).

The first patient was a 29 year old man who had had a left anterior pontine lacunar infarct 33 months previously (fig 4A). He initially presented with a right upper motor neurone facial palsy and a right sided hemiplegia. There was no history of vertigo and ocular movements were normal. Lower limb sensation was intact during clinical testing in the immediate period after stroke and when tested at the time of our study. The response to GVS was similar to that seen after MCA stroke (fig 5A and B). The postural sway in response to GVS was enhanced, whereas the individual lateral reaction forces were asymmetrical, as highlighted by an asymmetry index of -0.80 . The size of the MEP amplitude was also decreased after TMS (asymmetry index, -0.12 ) and delayed by $10.5 \mathrm{~ms}$. The similarity between this patient and those with MCA stroke is further highlighted by the similar relation between the relative delay in the TMS response and the GVS asymmetry index (star symbol in fig 3C).

The second patient was a 73 year old woman who presented with a five year history of left leg paresis and increasing difficulty in walking. At the time of our study she was able to walk short distances $(\sim 10 \mathrm{~m})$ with a frame and supervision. She presented with left lower limb weakness (3$4 / 5)$, whereas upper limb strength was only mildly affected $(4+/ 5)$. Lower and upper limb sensation was normal, reflexes were symmetrical, and there was a left extensor plantar response. Hearing was reduced on the left, and there were no other signs of cranial nerve or cerebellar involvement. EMG nerve conduction studies showed no signs of a motor neuropathy or radiculopathy, and MRI of the spine showed no signs of cord or root compression. MRI and intracranial magnetic resonance angiography revealed a right vertebral artery aneurysm compressing the right pyramid in the rostral medulla (fig 4B).

In this patient, the size of the MEP amplitude decreased after TMS (asymmetry index, -0.68 ), although it was not delayed. The response to GVS was assessed with the eyes open, visual acuity being already greatly decreased by the presence of bilateral dense cataracts. The response to GVS was approximately symmetrical, with the paretic leg contributing the greater response (asymmetry index, +0.16; fig 5C).

\section{DISCUSSION}

The main finding of our study was an abnormal inter-leg response asymmetry to GVS in the stroke group. We characterised the response primarily by measuring the change in the lateral force produced through each leg, rather than the electromyographic response of individual muscles. There are some advantages to this approach because the force represents the net result of all the distributed muscle activities and can readily be related to function. Furthermore, the signal to noise ratio of the force response is superior to that of an EMG response, requiring the averaging of far fewer trials to obtain a reasonable response estimate, which is an important consideration when studying disabled subjects. The reaction forces were measured over a short period from 320-500 ms after the onset of the stimulus. This initial period was before appreciable movements of the head and axial skeleton. Therefore, the initial response is probably purely vestibular in origin, and uncomplicated by movement related afferent feedback or the passive biomechanical effects of positional change.

The use of monaural stimulation allowed us to investigate whether there was any difference in response processing when the stimulus was applied either ipsilateral or contralateral to the lesion. In addition, by varying the polarity of stimulation we were able to distinguish whether there were any differences in the response when the subject swayed towards or away from the side of the lesion. However, regardless of the side of stimulation or the subsequent sway direction, we found no difference in the pattern or amplitude of the inter-leg response asymmetry. Thus, stroke was associated with a lateralised deficit in the motor output stage of vestibular processing, rather than in the sensory or spatial processing stages. 


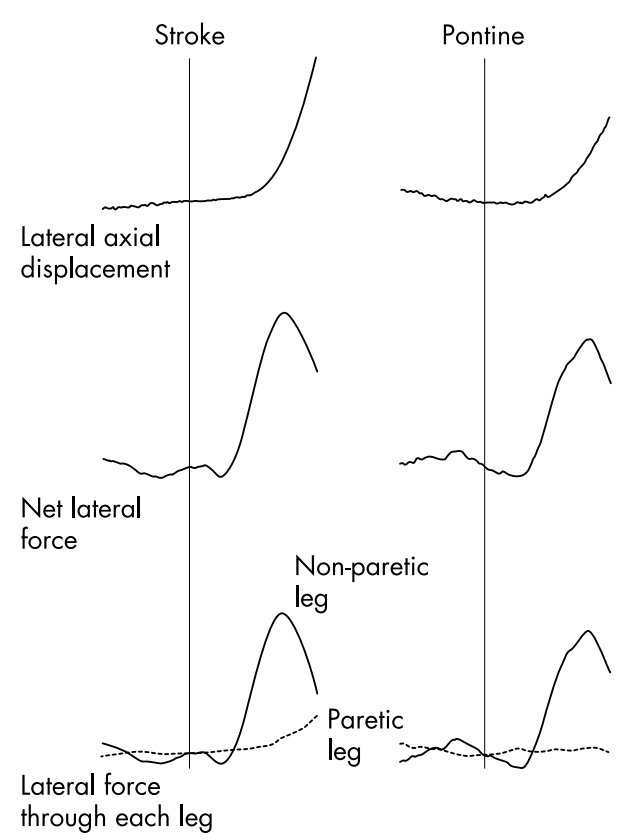

C

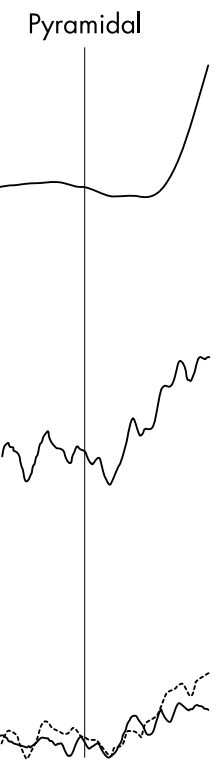

D

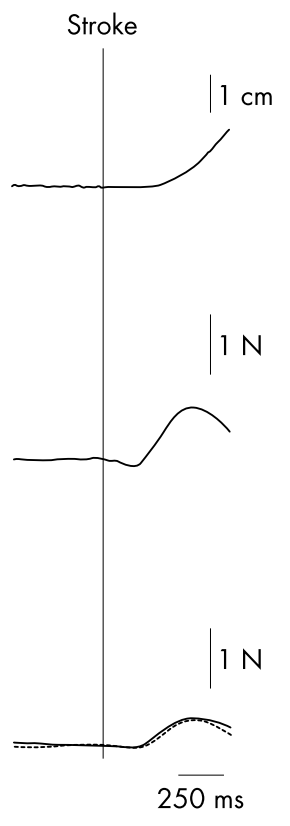

Figure 5 Comparison of galvanic vestibular stimulation response symmetry after lesions at successive levels of the neuraxis. (A) Grand average response of 16 patients with a middle cerebral artery stroke. (B) Response of subject 1 with an anterior pontine lesion. (C) Response of subject 2 with a compression of the right pyramid. (D) Grand average response of 10 control subjects. All responses are the average of the four stimulation conditions. The vertical line indicates stimulus onset.

Previous studies of standing balance after stroke have found deficits in the motor control of the paretic leg. ${ }^{3-7}$ In our present study, there was no significant difference in the response amplitude of the stroke group's paretic limbs compared with the healthy limbs of control subjects. The obvious difference was in the behaviour of the non-paretic leg, which appeared to over respond. However, there are two factors that complicate this interpretation. First, the feet were placed $5 \mathrm{~cm}$ apart to enable the stroke subjects to stand independently with their eyes closed. In control subjects, relatively small increases in stance width, compared with when the feet are together, have the effect of greatly reducing the amplitude of the response. ${ }^{23}{ }^{24}$ Under these circumstances, it may be difficult to establish a reduction in paretic limb response size when the control response is already very small. On top of this, the stroke group's baseline body sway was greater than the control group, indicating that they were less stable. It is well established that conditions that increase instability and background body sway, such as the removal of visual or tactile cues, ${ }^{16}$ or neurological disease, ${ }^{25}$ are associated with enlarged responses to GVS. Again, the effect of this would be to mask any differences between an underresponding paretic limb and a healthy limb, while at the same time accentuating the apparent over-responsiveness of the non-paretic limb. What is clear, however, is that the overall whole body response was abnormally asymmetrical in the stroke group; if a small response was seen in a limb of a control subject then it occurred symmetrically in both legs.

Our results suggest that a chronic balance deficit can arise as a consequence of stroke. The two legs no longer contribute equally to the balance process, even when they are taking an equal amount of body weight. The responsibility for balance control seems to be shifted away from the paretic leg and towards the relatively unaffected leg. The correlation data suggest that this phenomenon is not simply a reflection of muscle weakness or sensory loss contralateral to the lesion. However, we did find that the GVS asymmetry index correlated with the relative delay in MEP onset latency after
TMS. Experimental models of stroke in the monkey suggest that the delay in MEP onset predominately reflects the number of fibres that have been damaged. Ischaemia causes preferential damage of the larger, faster conducting corticospinal neurones, resulting in a population with an overall reduced conduction velocity and a delay in onset latency. ${ }^{20} 26$ Thus, the relative delay in TMS onset after stroke may serve as an indicator of the degree of damage to motor cortical output neurones.

It has previously been suggested that the balance response to GVS may be mediated via brainstem nuclei. ${ }^{16}{ }^{17}$ We suggest that MCA stroke produces its effect by disrupting projections from the cortex of one hemisphere to these brainstem motor centres. The results of the two patients with discrete pyramidal lesions support this view. It predicts that such corticobulbar projections may be affected by a lesion rostral to the lower pons, but not by a lesion in the medulla below the level of the vestibular nuclei, which was found to be the case. Thus, for the patient with the pontine lesion, the responses to both GVS and TMS were asymmetrical to a degree similar to that seen after an MCA lesion. In contrast, for the patient with the medullary lesion, the response to GVS was symmetrical, and of a similar magnitude to that seen in control subjects, whereas the TMS response was asymmetrical.

The proposed corticobulbar projections could be either collaterals of the corticospinal tract or separate neurones that lie spatially close to it, explaining the association between the GVS evoked asymmetry and the degree of corticospinal tract damage. Such a spatial proximity between the corticospinal tract and corticobulbar projections is seen for example in the projections to the facial nucleus. ${ }^{27}$ Corticobulbar connections from the cortex to the reticular or vestibular nuclei have been found in non-human species. For example, in the cat and primate, collaterals from the corticospinal tract, in addition to separate pathways, arise bilaterally from layer $\mathrm{V}$ of the primary and premotor cortices and project to the pontomedullary reticular formation. ${ }^{28-31}$ Similarly, cortical projections 
from the contralateral premotor cortex to the lateral vestibular nucleus have been identified in old and new world monkeys, ${ }^{32} 33$ and are thought to modulate vestibulomotor reflex arcs. ${ }^{32}$ Cortical projections to the vestibular nuclei also arise from the parietal lobe. ${ }^{32}$ Lesions to the parietal multisensory cortex can result in deficits in balance and the perception of verticality. ${ }^{34-38}$ Therefore, these projections may also play a role in the vestibular control of balance, in addition to their known role in the control of ocular movements. ${ }^{39}$ The deficits in balance and verticality perception seen after parietal lesions are often associated with signs of visuo-spatial neglect, ${ }^{34-36}$ a symptom that was absent in our present cohort of patients. Furthermore, none of our patients showed signs of pushing away from the non-paretic side, a symptom often associated with acute temporoparietal lesions. ${ }^{34}{ }^{37}$ Nevertheless, the patients with large GVS asymmetry indices had lesions that affected both the frontal and parietal cortex (table 2), so it is possible that these proposed corticobulbar projections could arise from either the frontal or parietal lobes.

Disruption of the proposed corticobulbar connections could indirectly result in a reduced response on the paretic side. Reciprocal connections between brain stem centres each side of the neuraxis could explain the response asymmetry seen between the two sides. For example, the vestibular nuclei are reciprocally connected via inhibitory commissural connections. Here, a lesion resulting in a decrease in activity on one side could disinhibit the opposite side and thereby increase its excitability. Such asymmetries are seen after a unilateral vestibular nerve lesion. ${ }^{40}$ Therefore, this proposal provides a possible explanation for the asymmetrical response to GVS seen in the stroke group. Because the degree of asymmetry would depend upon the extent of the damage to cortical output pathways, it may also explain the association between the GVS evoked response asymmetry and the TMS response delay.

There are other possible pathophysiological mechanisms that could explain the asymmetrical GVS response. Vestibular stimuli directly activate an interconnected circuit of cortical areas within the parietal, temporal, and frontal lobes. ${ }^{41-43}$ Therefore, the postural response evoked by GVS could be mediated through a transcortical pathway, whose output is via the corticospinal tract. This could explain the GVS asymmetry seen with an MCA lesion and the association with corticospinal tract damage. Another possibility is that the spinal cord is altered by the loss of corticospinal inputs and so responds abnormally to activity in other descending pathways. However, the finding of a symmetrical response to GVS after a corticospinal tract lesion at the level of the medulla argues against these possibilities.

In conclusion, we have shown that MCA stroke disrupts the vestibular channel of balance control. We propose that stroke may produce this effect by interrupting corticobulbar modulation of brainstem balance centres. We assume that the neurones responsible lie spatially close to the corticospinal tract, such that damage to the corticospinal tract acts as a marker of damage to the proposed corticobulbar pathway. The present data do not indicate whether the vestibular motor changes are immediate consequences of stroke or are the result of longer term compensatory processes. This can only be answered by studying subjects at much shorter intervals after stroke. Nevertheless, the asymmetry that we have described may contribute to the asymmetries in standing balance often reported in stroke patients.

\section{ACKNOWLEDGEMENTS}

We would like to thank Dr P Brown and Dr P Rudge for their help with patient recruitment.

\section{Authors' affiliations}

J F Marsden, B L Day, MRC Human Movement Group, Sobell

Department of Motor Neuroscience and Movement Disorders, Institute of Neurology, Queen Square, London WCIN 3BG, UK

D E Playford, J F Marsden, Neurorehabilitation and Therapy Services Department, National Hospital for Neurology and Neurosurgery, Queen Square, London WCIN 3BG, UK

Competing interests: none declared

\section{REFERENCES}

1 Bohannon RW, Larkin PA. Lower extremity weight bearing under various standing conditions in independently ambulatory patients with hemiparesis. Phys Ther 1985;65:1323-5.

2 Mizrahi J, Solzi P, Ring $\mathrm{H}$, et al. Postural stability in stroke patients: vectorial expression of asymmetry, sway activity and relative sequence of reactive forces. Med Biol Eng Comput 1989;27:181-90.

3 Di Fabio RP. Adaptation of postural stability following stroke. Top Stroke Rehabil 1997;3:62-75.

4 Di Fabio RP. Lower leg extremity antagonist muscle response following standing perturbation in subjects with cerebrovascular disease. Brain Res 1987;406:43-51

5 Di Fabio RP, Badke MB, Duncan PW. Adapting human postural reflexes following localised cerebrovascular lesion: analysis of bilateral long latency responses. Brain Res 1986;363:257-64.

6 Di Fabio RP, Badke MB. Influence of cerebrovascular accident on elongated and passively shortened muscle responses after forward sway. Phys Ther 1988;68:1215-20.

7 Kirker SGB, Jenner JR, Simpson DS, et al. Changing patterns of postural hip muscle activity during recovery from stroke. Clin Rehabil 2000;14:618-26.

8 Horak FB, Esselman P, Lynch MK. The effects of movement velocity, mass displaced and task certainty on associated postural adjustments made by normal and hemiplegic subjects. J Neurol Neurosurg Psychiatry 1984:47:1020-8.

9 Rogers MW, Hedman LD, Pai Y-C. Kinetic analysis of dynamic transitions in stance support accompanying voluntary leg flexion movements in hemiparetic adults. Arch Phys Med Rehabil 1993;74:19-25.

10 Garland SJ, Stevenson TJ, Ivanova T. Postural responses to unilateral arm perturbation in young, elderly and hemiplegic subjects. Arch Phys Med Rehabil 1997;78:1072-7.

11 Palmer E, Downes L, Ashby P. Associated postural adjustments are impaired by a lesion of the cortex. Neurology 1996;46:471-5.

12 Lowenstein $\mathrm{O}$. The effect of galvanic polarization on the impulse discharge from sense endings in the isolated labyrinth of the thornback ray (Raja clavata). J Physiol (Lond) 1955;127:104-17.

13 Goldberg JM, Fernandez C, Smith CE. Responses of vestibular nerve afferents in the squirrel monkey to externally applied galvanic currents. Brain Res 1982;252:156-60.

14 Courion JH, Precht W, Sutherling WW. Vestibular nerve and nuclei unit responses and eye movement responses to repetitive galvanic vestibular stimulation of the labyrinth in the rat. Exp Brain Res 1987;66:41-8.

15 Severac-Cauquil A, Day BL. Galvanic vestibular stimulation modulates voluntary movement of the human upper body. J Physiol (Lond) 1998;513:611-19.

16 Britton TC, Day BL, Rothwell J, et al. Postural electromyographic responses in the arm and leg following galvanic vestibular stimulation in man. J Physiol (Lond) 1993;94:143-51.

17 Muto N, Shinomiya K, Komori H, et al. Spinal cord monitoring of ventral funiculus function. Analysis of spinal field potentials after galvanic vestibular stimulation. Spine 1995;20:2429-34.

18 Watson SRD, Colebatch JG. EMG responses in the soleus muscles evoked by unipolar galvanic vestibular stimulation. Electroencephalogr Clin Neurophysiol 1997; 105:476-83.

19 Marsden JF, Day BL. Bilateral lower limb responses evoked by unilateral vestibular stimulation in humans. In: Lord SR, Menz HB, eds. Sydney: Proceedings of the International Society for Postural and Gait Research XVth conference, 2003:115.

20 Thompson PD, Day BL, Rothwell J, et al. The interpretation of electromyographic responses to electrical stimulation of the motor cortex in diseases of the upper motor neurone. J Neurol Sci 1987;80:91-110.

21 Berardelli A, Inghilleri $M$, Cruccu $G$, et al. Electrical and magnetic transcranial stimulation in patients with corticospinal damage due to stroke or motor neurone disease. Electroencephalogr Clin Neurophysiol 1991;81:389-96.

22 Medical Research Council. Aids to the examination of the peripheral nervous system. London: Bailliere Tindall, 1986.

23 Day BL, Severac-Cauquil A, Bartolomei L, et al. Human body-segment tilts induced by galvanic stimulation: a vestibularly driven balance protection mechanism. J Physiol (Lond) 1997;500:661-72.

24 Welgampola MS, Colebatch JG. Vestibulospinal reflexes: quantitative effects of sensory feedback and postural task. Exp Brain Res 2001;139:345-53.

25 Pastor MA, Day BL, Marsden CD. Vestibular induced postural responses in Parkinson's disease. Brain 1993;116:1177-90.

26 Branston NM, Bentivoglio P, Momma F, et al. Changes in pyramidal tract conduction with experimental brain-stem ischaemia in the monkey. Electroencephalogr Clin Neurophysiol 1987;69:469-75. 
27 Terao S, Miura N, Takeda A, et al. Course and distribution of facial corticobulbar tract fibers in the lower brain stem. J Neurol Neurosurg Psychiatry 2000;69:262-5

28 Keizer K, Kuypers HGJM. Distribution of corticospinal neurones with collaterals to the lower brain stem reticular formation in the monkey (Macaca fascicularis). Exp Brain Res 1989;74:311-18.

29 Kably B, Drew T. Corticoreticular pathways in the cat. I. Projection patterns and collateralisation. J Neurophysiol 1998;80:389-405.

30 Lamas JA, Martinez L, Candeo A. Pericruciate fibers to the red nucleus and to the medial bulbar reticular formation. Neuroscience 1994;62:115-24.

31 Keizer K, Kuypers HGJM. Distribution of corticospinal neurons with collaterals to lower brainstem reticular formation in cat. Exp Brain Res 1984;54:107-20.

32 Akbarian S, Grusser O-J, Guldin WO. Corticofugal connections between the cerebral cortex and brainstem vestibular nuclei in the macaque monkey. J Comp Neurol 1994:339:421-37.

33 Akbarian S, Grusser O-J, Guldin WO. Corticofugal projections to the vestibular nuclei in squirrel monkeys - further evidence of multiple cortical vestibular fields. J Comp Neurol 1993;332:89-104.

34 Perennou DA, Amblard B, Laassel EM, et al. Understanding the pusher behavior of some stroke patients with spatial deficits. Arch Phys Med Rehabil 2002;83:570-5.
35 Perennou DA, Leblond C, Amblard B, et al. The polymodal sensory cortex is crucial for controlling lateral postural stability: evidence from stroke patients. Brain Res Bull 2000;53:359-65.

36 Yelnik J, Lebreton F, Bonan I, et al. Perception of verticality after recent cerebral hemispheric stroke. Stroke 2002;33:2247-53.

37 Karnath HO, Ferber S, Dichgans J. Neural representation of postural control in humans. Proc Natl Acad Sci U S A 2000;97:13931-6.

38 Brandt T, Dieterich M. The vestibular cortex. Its locations, functions, and disorders. Ann N Y Acad Sci 2004:871:293-312.

39 Fukushima K. Corticovestibular interactions: anatomy, electrophysiology, and functional considerations. Exp Brain Res 1997;117:1-6.

40 Curthoys IS, Halmagyi GM. Vestibular compensation: a review of the oculomotor, neural and clinical consequences of unilateral vestibular loss. J Vestib Res 1995:5:67-107.

41 Lobel E, Kleine JF, Bihan D, et al. Cortical areas activated by bilateral galvanic vestibular stimulation. Ann N Y Acad Sci 1998;8871:313-23.

42 Bucher SF, Dieterich $M$, Wiesmann $M$, et al. Cerebral functional magnetic resonance imaging of vestibular, auditory, and nociceptive areas during galvanic stimulation. Ann Neurol 1998:44:120-5.

43 Guldin WO, Grusser O-J. Is there a vestibular cortex? Trends Neurosci 1998;21:254-9.

\section{$\mathrm{ECHO}$}

\section{Cannabis may cause psychosis in young people}

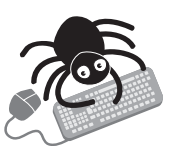

Please visit the Journal of

Neurology, Neurosurgery, and Psychiatry website [www.jnnp. com] for a link to the full text of this article.
A large prospective population based study in young people has confirmed that using cannabis risks psychosis developing later-greatly for those susceptible to psychosis and moderately for others. It also provides evidence that using cannabis might cause psychosis and is not just a consequence of it.

Young people using cannabis at baseline in the study were more likely to have psychotic symptoms four years later (odds ratio (OR) 1.67), after adjustment for a slew of confounding factors. The likelihood of having any psychotic symptoms rose with frequency of use in a dose-response way, from 0.99 for use less than once a month to 2.23 for almost daily use. The adjusted difference in risk of psychosis with cannabis use was $23.8 \%$ for young people predisposed to psychosis but $5.6 \%$ for the rest. Predisposition to psychosis at baseline, however, did not significantly affect use of cannabis during the next four years (OR $1.42)$.

The study analysed data on 2437 young people aged 14-24 years who were part of the random regional representative population sample in the prospective early developmental stages of psychopathology study (EDSP) in Munich, Germany.

Whether using cannabis causes psychosis has been disputed, some arguing that predisposition to psychosis may be the driving force to taking up the drug, rather than cannabis causing psychosis to be expressed. This-the first prospective study to research the issue-suggests that cannabis may be the culprit.

$\Delta$ Henquet $C$, et al. BMJ 2005;333:11-14. 